\title{
ÁCIDO CIANÍDRICO EM ALGUMAS VARIEDADES DE MANDIOCA
}

Francisco Alves Correia

\section{INTRODUÇÃO}

Em continuação aos trabalhos que vêm sendo levados a efeito pela Seç̧ão de Tecnologia Agrícola do Instituto Agronômico do Estado de São Paulo, e referentes a Manihot utilissima Pohl, iniciamos uma série de análises visando um conhecimento mais amplo sôbre o teor em ácido cianídrico (HCN) nas raízes dessa euforbiácea; êsse estudo poderá ser de menor importância para as grandes fábricas de fécula ou de farinha de mandioca, porquanto a quase totalidade do HCN é separada durante a série de lavagens, secagem ou, no segundo caso, prensagem e posterior torração do produto. Não acontece o mesmo quando usada na alimentação dos animais domésticos, conforme será visto mais adiante.

Com o aumento do número de fecularias no Estado e ainda a adaptação das já existentes para maior capacidade, resultou maior incremento na cultura da mandioca, principalmente nas proximidades das fábricas ; havendo sensível falta de ramas para o plantio, o preço destas subiu, daí advindo grande movimento de ramas de uma região para outra do Estado, sem conhecimento perfeito, por parte dos compradores, das qualidades da planta - mãe. Um estudo, ainda que em caráter preliminar, dessas variedades, será de interêsse, pois serão elas usadas tanto na alimentação humana como na dos animais, não só nas cidades, mas, e principalmente, entre os nossos homens do campo. No tocante ao uso na alimentação humana, o perigo se torna quase inexistente, tendo-se em vista que as raízes são descascadas e sofrem aquecimento prolongado durante o seu preparo ; por outro lado, na alimentação dos animais domésticos, o perigo é maior, pois elas não passam pelo tratamento acima, levando, destarte, o conteúdo quase total de HCN para o organismo do animal.

\section{DESCRIÇÃO DO METODO}

Todos os métodos por nós examinados tinham por base a libertação do CN do complexo glucocianogênico da mandioca, por digestão simples com água ou por meio de um ácido forte.

O método escolhido foi o de Liebig (3), que consta do seguinte : em um balão colocam-se $50 \mathrm{gr}$ (cilindro central) ou $20 \mathrm{gr}$ (casca) do material 
ralado, juntam-se 300 a 400 cc de água e deixa-se em digestão durante uma e meia a duas horas ; coloca-se o balão no aparelho e deixa-se distilar por vinte minutos, recebendo-se em meio alcalino ; finda a distilação, titula-se com nitrato de prata, usando-se como indicador o iodureto de potássio (KI).

\section{RESULTADOS DAS ANĀLISES}

Iniciamos os nossos trabalhos com uma variedade que foi recentemente distribuída pela Seç̧ão de Raízes e Tubérculos, do Instituto Agronômico, a título experimental, para os diversos Campos de Aumento do Estado. Trata-se da variedade 454, em alguns lugares chamada de Guaxupé, e cujos caraterísticos culinários foram, sob certos aspectos, superiores aos da conhecida "Vassourinha", tão difundida pelo nosso interior.

Feita a dosagem de ácido cianídrico acusou esta os resultados médios seguintes :

Q U A D R O 1

\begin{tabular}{|c|c|c|}
\hline \multirow{2}{*}{$\mathrm{HCN}$} & \multicolumn{2}{|c|}{ VARIEDADES } \\
\hline & 454 & Vassourinha \\
\hline $\begin{array}{l}\text { No cilindro central } \ldots \\
\mathrm{Na} \text { casca } \ldots \ldots \ldots\end{array}$ & $\begin{array}{l}0,001 \% \\
0,028 \%\end{array}$ & $\begin{array}{l}0,002 \% \\
0,053 \%\end{array}$ \\
\hline
\end{tabular}

Trabalhando com quatro variedades, gentilmente cedidas pelo professor Carlos Teixeira Mendes, da Escola Superior de Agricultura "Luiz de Queiroz", de Piracicaba, sendo as três primeiras importadas da Bahia, em 1939, e lá consideradas como das mais venenosas existentes, obtivemos resultados bastante concordantes, tendo-se em vista a regularidade das repetições. (Quadro 2).

\section{Q U A D R O 2}

MANDIOCAS COM 16 MESES, COLHIDAS EM JUNHO DE 1946

\begin{tabular}{|c|c|c|c|c|}
\hline \multirow{3}{*}{\multicolumn{2}{|c|}{ VA R I E A D E S }} & \multicolumn{3}{|c|}{$\mathrm{H} \mathrm{C} \mathrm{N}$} \\
\hline & & \multicolumn{3}{|c|}{ D I G E S T A O } \\
\hline & & 20 horas & 44 horas & 68 horas \\
\hline Preta da Bahia & $\left\{\begin{array}{l}\text { Cil. central....... } \\
\text { Casca............. }\end{array}\right.$ & $\begin{array}{l}0,005 \% \\
0,050 \%\end{array}$ & $\begin{array}{l}0,004 \% \\
0,054 \%\end{array}$ & $\begin{array}{l}0,003 \% \\
0,050 \%\end{array}$ \\
\hline Mata Negro & $\left\{\begin{array}{l}\text { Cil. central....... } \\
\text { Casca........... }\end{array}\right.$ & $\begin{array}{l}0,016 \% \\
0,123 \%\end{array}$ & $\begin{array}{l}0,016 \% \\
0,123 \%\end{array}$ & $\begin{array}{l}0,016 \% \\
0,117 \%\end{array}$ \\
\hline Preta de Suruí & $\left\{\begin{array}{l}\text { Cil. central....... } \\
\text { Casca............ }\end{array}\right.$ & $\begin{array}{l}0,016 \% \\
0,174 \%\end{array}$ & $\begin{array}{l}0,014 \% \\
0,179 \%\end{array}$ & $\begin{array}{l}0,018 \% \\
0,178 \%\end{array}$ \\
\hline Vassourinha & $\left\{\begin{array}{l}\text { Cil. central. } \\
\text { Casca...... }\end{array}\right.$ & $\begin{array}{l}0,002 \% \\
0,055 \%\end{array}$ & $\begin{array}{l}0,001 \% \\
0,051 \%\end{array}$ & $\begin{array}{l}0,002 \% \\
0,054 \%\end{array}$ \\
\hline
\end{tabular}

(*) - Amostras da variedade 454, com 7 meses de idade, cultivadas na Fazenda Mato Dentro, do Instituto Biológico do Estado de São Paulo. Análises 16.934/41 do Instituto Agronômico. 
Para comprovar que a totalidade do HCN foi distilada nos primeiros vinte minutos, foi feita a seguinte prova : retirado o balão Erlenmeyer, com o distilado, foi êle substitúdo por outro balão contendo a mesma quantidade de meio alcalino, reiniciando-se a distilação durante mais vinte minutos: Os resultados expressos no quadro 3 demonstram que a quantidade de HCN ainda distilada teve valor insignificante.

Q U A D R O 3

\begin{tabular}{|c|c|c|c|}
\hline \multirow{3}{*}{ VA I I D A D E S } & \multicolumn{3}{|c|}{$\mathrm{HCN}$} \\
\hline & \multicolumn{3}{|c|}{ D I G E S T A O } \\
\hline & 20 horas & 44 horas & 68 horas \\
\hline$\dot{\text { Preta da Bahia } \ldots \ldots}\left\{\begin{array}{l}\text { Cil. central . . . . } \\
\text { Casca . . . . . . }\end{array}\right.$ & $\begin{array}{l}\mathbf{0 , 0 0 1} \% \\
0,001 \%\end{array}$ & $\begin{array}{l}\mathbf{0 , 0 0 1} \% \\
\mathbf{0 , 0 0 2} \%\end{array}$ & $\begin{array}{l}\text { traços } \\
0,001 \%\end{array}$ \\
\hline$\left\{\begin{array}{l}\text { Cil. central . . . } \\
\text { Casca } \ldots \ldots \ldots\end{array}\right.$ & $\begin{array}{l}0,001 \% \\
0,003 \%\end{array}$ & $\begin{array}{l}\mathbf{0 , 0 0 1} \% \\
\mathbf{0 , 0 0 1} \%\end{array}$ & $\begin{array}{l}0,001 \% \\
0,003 \%\end{array}$ \\
\hline$\ldots\left\{\begin{array}{l}\text { Cil. central . . . . . . . } \\
\text { Casca } \ldots\end{array}\right.$ & $\begin{array}{l}0,001 \% \\
0,004 \%\end{array}$ & $\begin{array}{l}0,001 \% \\
0,003 \%\end{array}$ & $\begin{array}{l}0,001 \% \\
0,003 \%\end{array}$ \\
\hline$\left\{\begin{array}{l}\text { Cil. central. } \\
\text { Casca...... }\end{array}\right.$ & $\begin{array}{l}\text { traços } \\
0,003 \%\end{array}$ & $\begin{array}{l}\text { traços } \\
0,001 \%\end{array}$ & $\begin{array}{l}\text { traços } \\
\text { traços }\end{array}$ \\
\hline
\end{tabular}

Ainda para que não pairassem dúvidas a êsse respeito, foi feita a seguinte prova com duas outras variedades : finda a primeira distilação, o balão contendo o material em exame foi retirado, tapado e deixado de lado até resfriamento completo ; por fim, foram juntados $10 \mathrm{cc}$ de ácido forte, ficando em digestão por mais 24 horas ; após, repetiu-se a distilação por vinte minutos, sendo os resultados os constantes do quadro 4.

Q U A D R O 4

\section{A R I E D A D E S}

$\mathrm{H} \mathrm{C} \mathrm{N}$

\begin{tabular}{|c|c|c|}
\hline \multirow{2}{*}{$V A R I E$} & \multirow{2}{*}{ Casca } & \multirow{2}{*}{ Cil. central } \\
\hline & & \\
\hline Brava de Itu $\ldots \ldots \ldots\left\{\begin{array}{l}1 .^{\mathrm{a}} \text { dist. } \\
2 .^{\mathrm{a}} \text { dist. }\end{array}\right.$ & $\begin{array}{l}\mathbf{0 , 0 7 7 0} \% \\
0,0002 \%\end{array}$ & $\begin{array}{l}\mathbf{0 , 0 1 8 0} \% \\
0,0005 \%\end{array}$ \\
\hline Roxa de Galho..... $\left\{\begin{array}{l}1 .{ }^{\mathrm{a}} \text { dist. } \\
2 .^{\mathbf{a}} \text { dist. }\end{array}\right.$ & $\begin{array}{l}0,0660 \% \\
0,0002 \%\end{array}$ & $\begin{array}{l}0,0040 \% \\
0,0002 \%\end{array}$ \\
\hline
\end{tabular}

Em vista de têrmos, por motivos alheios à nossa vontade, perdido as provas de mais rápida digestão na água, consideramos os resultados do quadro 2 como preliminares, sendo as análises repetidas com menor: 
tempo de digestão; deixamos, então, a amostra ralada em digestão por $11 / 2,31 / 2$ e $51 / 2$ horas, respectivamente. Pelo quadro 5 notamos que, de maneira geral, não houve vantagem em deixar-se por mais que $11 / 2$ horas em digestão na água.

\section{Q U A D R O 5}

PLANTAS COM 22 MESES, COLHIDAS EM JANEIRO DE 1947

\begin{tabular}{|c|c|c|c|}
\hline \multirow{3}{*}{ "S E E D L I N G" } & \multicolumn{3}{|c|}{$\mathrm{H} \mathrm{C} \mathrm{N}$} \\
\hline & \multicolumn{3}{|c|}{$D I G E S T A O$} \\
\hline & $11 / 2$ horas & $31 / 2$ horas & $51 / 2$ horas \\
\hline$\ldots\left\{\begin{array}{l}\text { Cil. central. } \\
\text { Casca...... }\end{array}\right.$ & $\begin{array}{l}0,006 \% \\
0,057 \%\end{array}$ & $\begin{array}{l}0,005 \% \\
0,070 \%\end{array}$ & $\begin{array}{l}0,008 \% \\
0,070 \%\end{array}$ \\
\hline$\ldots\left\{\begin{array}{l}\text { Cil. central. } \\
\text { Casca....... }\end{array}\right.$ & $\begin{array}{l}0,001 \% \\
0,058 \%\end{array}$ & $\begin{array}{l}0,003 \% \\
0,062 \%\end{array}$ & $\begin{array}{l}0,003 \% \\
0,062 \%\end{array}$ \\
\hline$\cdots\left\{\begin{array}{l}\text { Cil. central. } \\
\text { Casca....... }\end{array}\right.$ & $\begin{array}{l}0,001 \% \\
0,046 \%\end{array}$ & $\begin{array}{l}0,001 \% \\
0,046 \%\end{array}$ & $\begin{array}{l}0,002 \% \\
0,043 \%\end{array}$ \\
\hline
\end{tabular}

Outras variedades cultivadas na Estação Experimental Central, do Instituto Agronômico, nos deram resultados bastante interessantes (Quadro 6), porquanto, da mesma maneira que os quadros 1,2 e 5 , verificamos que a percentagem de $\mathrm{HCN}$ na casca da mandioca é muito mais elevada que no cilindro central, e, levando-se em conta a percentagem de casca e cilindro central na mandioca (resultados médios da variedade Vassourinha em oito meses de observação e que, segundo Godói (2) é de 17,06\% e 80,00\%) e os resultados médios do quadro 6, poderemos concluir que a casca contém $60 \%$ do HCN total contido na mandioca, ficando o cilindro central com os $40 \%$ restantes, e isso porque :

em 100 gr de mandioca há 17,06 gr de casca

em 100 gr de casca há 0,072 gr de $\mathrm{HCN}$, ou melhor, essa quantidade de HCN como resultante do complexo cianogênico (Média do quadro 6).

Donde :

em 100 gr de mandioca há $\frac{17,06 \times 0,072}{100}=0,012$ gr de $\mathrm{HCN}$.

Aplicando-se o mesmo cálculo para o cilindro central, encontraremos 0,008 gr de HCN.

HCN total em $100 \mathrm{gr}$ de mandioca:

$$
0,012+0,008=0,020 \mathrm{gr}
$$


No caso da casca, teremos:

$$
\frac{0,012 \times 100}{0,020}=60
$$

e para o cilindro central :

$$
\frac{0,008 \times 100}{0,020}=40 \text {. }
$$

Pelo exposto, conclui-se que, a despeito da maior percentagem em que o cilindro central entra na composição da mandioca, ainda êle não poderá ser responsabilizado pela maior percentagem de HCN dessas raízes, cabendo a maior parcela à casca.

\section{Q U A D R O 6}

\begin{tabular}{|c|c|c|}
\hline \multirow{2}{*}{ VA R I E D D E S } & \multicolumn{2}{|c|}{$\mathrm{HC} \mathrm{N}$} \\
\hline & Casca & Cil. central \\
\hline 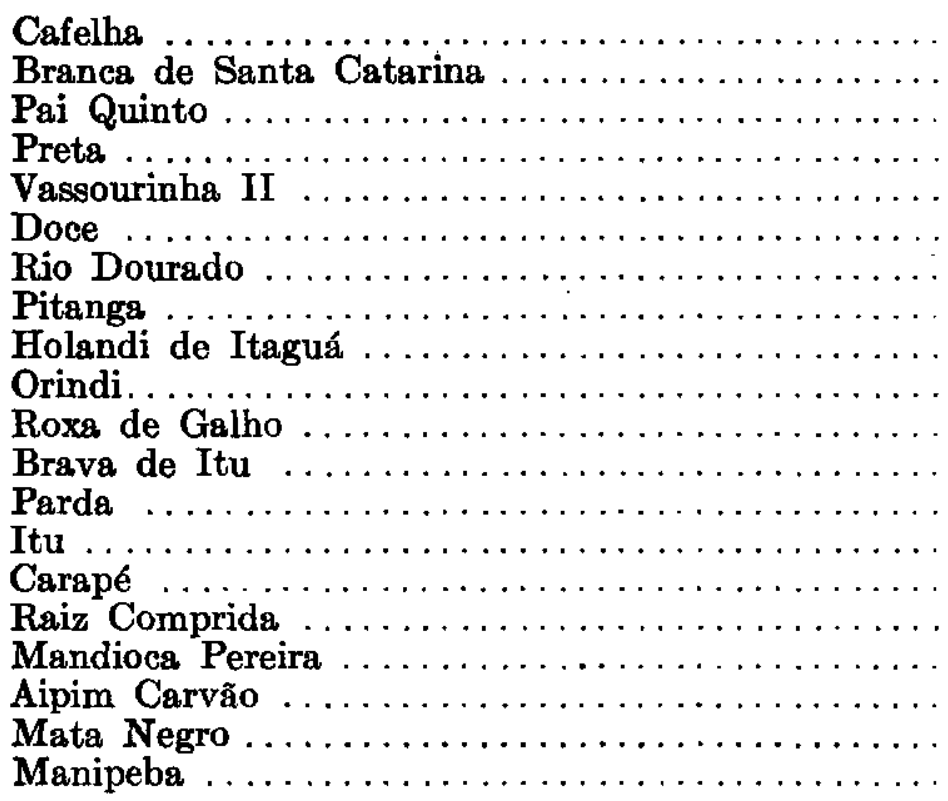 & $\begin{array}{l}0,053 \% \\
\mathbf{0 , 0 6 8} \% \\
0,128 \% \\
0,032 \% \\
0,042 \% \\
0,035 \% \\
0,086 \% \\
0,062 \% \\
0,054 \% \\
0,133 \% \\
0,066 \% \\
0,077 \% \\
0,043 \% \\
0,088 \% \\
0,119 \% \\
0,050 \% \\
0,097 \% \\
0,092 \% \\
0,066 \% \\
0,054 \%\end{array}$ & $\begin{array}{l}0,012 \% \\
0,005 \% \\
0,016 \% \\
0,010 \% \\
0,014 \% \\
0,004 \% \\
0,009 \% \\
0,025 \% \\
0,010 \% \\
0,008 \% \\
0,004 \% \\
0,018 \% \\
0,003 \% \\
0,031 \% \\
0,002 \% \\
0,016 \% \\
0,004 \% \\
0,004 \% \\
0,002 \% \\
0,012 \%\end{array}$ \\
\hline MÊDIAS . . . & $0,072 \%$ & $0,010 \%$ \\
\hline
\end{tabular}

MANDIOCAS COM 16 MESES, ARRANCADAS EM ABRIL DE 1946

Não encontramos uma relação constante entre as percentagens de HCN do cilindro central e da casca, nos ensaios até agora feitos, e, em vista. de não têrmos trabalhado com amostras da mesma espessura e comprimento, não poderemos afirmar que haja ou não essa relação. 


\section{RASPAS SECAS AO SOL}

Continuando os ensaios, foram examinadas amostras de raspas sêcas ao sol, sendo os resultados os constantes do quadro 7.

Uma parte das raízes foi despeliculada e ralada, sendo dessa massa retirada a amostra para a determinação de HCN. A outra parte foi transformada em raspas finas e exposta ao sol, durante três dias, para secagem e em seguida moída para a determinação de HCN.

Procuramos trabalhar sempre com amostras de mesma espessura para conseguir resultados mais homogêneos ; como se poderá notar, as raspas sêcas ao sol perdem a maior parte do seu ácido cianídrico.

Q U A D R O 7

\begin{tabular}{|c|c|c|c|c|}
\hline V A R I E D A D E S & $\begin{array}{l}\mathrm{HCN} \text { na } \\
\text { mandioca } \\
\text { inteira }\end{array}$ & $\begin{array}{l}\text { Umidade } \\
\text { perdida } \\
\text { ao sol }\end{array}$ & $\begin{array}{l}\text { HCN nas } \\
\text { raspas } \\
\text { sêcas }\end{array}$ & $\begin{array}{c}\text { Perda } \\
\text { de } \\
\mathrm{HCN}\end{array}$ \\
\hline 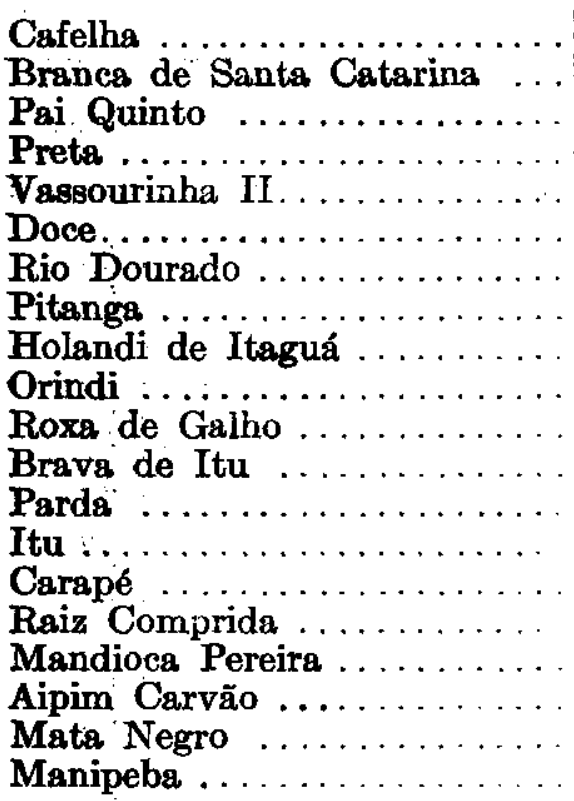 & $\begin{array}{l}\mathbf{0 , 0 2 2} \% \\
\mathbf{0 , 0 3 2} \% \\
\mathbf{0 , 0 4 3} \% \\
\mathbf{0 , 0 2 6} \% \\
\mathbf{0 , 0 1 7} \% \\
\mathbf{0 , 0 1 5} \% \\
\mathbf{0 , 0 2 4} \% \\
\mathbf{0 , 0 3 9} \% \\
\mathbf{0 , 0 4 0} \% \\
\mathbf{0 , 0 5 6} \% \\
\mathbf{0 , 0 2 2} \% \\
\mathbf{0 , 0 3 7} \% \\
\mathbf{0 , 0 1 4} \% \\
\mathbf{0 , 0 6 5} \% \\
\mathbf{0 , 0 2 0} \% \\
\mathbf{0 , 0 2 9} \% \\
\mathbf{0 , 0 1 7} \% \\
\mathbf{0 , 0 1 6} \% \\
\mathbf{0 , 0 2 3} \% \\
\mathbf{0 , 0 2 6} \%\end{array}$ & $\begin{array}{l}\mathbf{5 8 , 0} \% \\
\mathbf{6 0 , 0} \% \\
\mathbf{6 0 , 0} \% \\
\mathbf{6 4 , 0} \% \\
\mathbf{5 5 , 0} \% \\
\mathbf{6 0 , 0} \% \\
\mathbf{5 3 , 5} \% \\
\mathbf{5 9 , 0} \% \\
\mathbf{5 8 , 0} \% \\
\mathbf{5 9 , 0} \% \\
\mathbf{6 0 , 0} \% \\
\mathbf{6 0 , 0} \% \\
\mathbf{5 9 , 0} \% \\
\mathbf{5 2 , 0} \% \\
\mathbf{5 2 , 0} \% \\
\mathbf{5 8 , 0} \% \\
\mathbf{5 4 , 0} \% \\
\mathbf{5 2 , 0} \% \\
\mathbf{5 8 , 0} \% \\
\mathbf{5 8 , 0} \%\end{array}$ & $\begin{array}{l}\mathbf{0 , 0 2 4} \% \\
\mathbf{0 , 0 3 6} \% \\
\mathbf{0 , 0 3 7} \% \\
\mathbf{0 , 0 2 5} \% \\
0,006 \% \\
\mathbf{0 , 0 0 8} \% \\
\mathbf{0 , 0 1 6} \% \\
\mathbf{0 , 0 1 6} \% \\
\mathbf{0 , 0 1 6} \% \\
\mathbf{0 , 0 4 4} \% \\
\mathbf{0 , 0 1 1} \% \\
\mathbf{0 , 0 4 1} \% \\
\mathbf{0 , 0 0 9} \% \\
\mathbf{0 , 0 2 5} \% \\
\mathbf{0 , 0 1 4} \% \\
\mathbf{0 , 0 2 3} \% \\
\mathbf{0 , 0 1 0} \% \\
\mathbf{0 , 0 1 0} \% \\
\mathbf{0 , 0 0 8} \% \\
\mathbf{0 , 0 1 1} \%\end{array}$ & $\begin{array}{l}54 \\
\mathbf{5 6}\end{array}$ \\
\hline $\mathbf{M} \mathbf{E} \mathbf{D} \mathbf{A}$ & & & & $73 \%$ \\
\hline
\end{tabular}

\section{MANDIOCA COZIDA SEM CASCA}

A fim de verificar a influência da cocção sôbre a percentagem de HCN na mandioca, foram descascadas quatro amostras, cortadas em pedaços, de maneira semelhante ao que se faz nas cozinhas, e cozidas durante o tempo necessário para que elas amolecessem e ficassem em estado de serem comidas. 
Das amostras assim preparadas, uma parte ficou para a prova gustativa e a outra para ser analisada, quanto ao teor em HCN.

$O$ resultado da primeira prova foi negativo; as amostras em exame mantiveram-se duras e amargas, a despeito da cocção por duas ou mais horas, o que não acontece normalmente com as variedades Vassourinha, Tatu, Guaxupé, etc., que em poucos minutos amolecem.

0 resultado da análise é o constante do quadro 8 .

$$
\text { Q U A D R O } 8
$$

PLANTAS COM 14 MESES, COLHIDAS EM ABRIL DE 1946

\begin{tabular}{|c|c|c|}
\hline \multirow{2}{*}{ VAR I E D A DES } & \multicolumn{2}{|c|}{$\mathrm{HCN}$} \\
\hline & cilindro central & idem, cozido \\
\hline $\begin{array}{l}\text { Cambaia ...... } \\
\text { Rio Dourado } \\
\text { Roxa de Galho } \\
\text { Pitanga ....... }\end{array}$ & $\begin{array}{l}0,0110 \% \\
0,0050 \% \\
0,0110 \% \\
0,0150 \%\end{array}$ & $\begin{array}{l}0,0005 \% \\
0,0005 \% \\
0,0005 \% \\
0,0005 \%\end{array}$ \\
\hline
\end{tabular}

Pelos dados do quadro 8 se nota que a perda de HCN no material cozido foi pràticamente total, não oferecendo perigo de envenenamento, a não ser que haja outro princípio ativo nessas rázes e que cause êsses envenenamentos ; segundo Zehntner (4) já se conseguiu separar uma substância das raízes frescas, a qual, em doses mínimas, causa perturbaçбes gerais no sistema nervoso dos animais. O gôsto amargo e rijeza, porém, afastam qualquer possibilidade de aproveitamento dessas raízes na alimentação humana.

Trabalho semelhante e com conclusões idênticas foi feito por A. E. Collens (1), nos Estados Unidos, usando mandiocas tanto venenosas como de baixa percentagem de HCN. Segundo o autor acima, "se as raízes não foram convenientemente cozidas e forem deixadas dentro dágua por algum tempo, há a possibilidade de desenvolver-se uma pequena quantidade de ácido cianídrico".

\section{CONCLUSÕES}

$\mathrm{O}$ autor fêz uma série de análises em raízes de mandiocas mais comuns do Estado de São Paulo, concluindo que na casca está a maior percentagem de $\mathrm{HCN}$, sendo ela responsável por $60 \%$ do total de $\mathrm{HCN}$, por pêso de mandioca.

Diversas variedades comuns no Estado foram estudadas quanto ao teor em HCN, tanto na casca como no cilindro central.

As raspas expostas ao sol, para secagem, durante três dias, perdem, em média, $73 \%$ do total de ácido cianídrico.

A parte comestível da mandioca (cilindro central), cozida até o necessário amolecimento, cede pràticamente todo seu elemento tóxico de origem cianogênica. 


\section{LITERATURA CITADA}

1. Collens, A. E. Bitter and sweet cassava - hydrocyanic contents. Bul. Dept. Agr. Trinidad and Tobago 14 : 54-56. 1915.

2. Godói, Juvenal Mendes de. Em Fecularia e Amidonaria, 2.a Ed., pág. 23.

3. Treadwell, F. P. $E m$ Manuel de Chimie Analytique, vol. 2, pág. 662, 4.. Ed., 1934.

4. Zehntner, L. Em Estudo sôbre algumas variedades de mandiocas brasileiras. Soc. Nac. de Agr. Rio. Imprensa Inglesa, 1919. 